[Phys. Rev. E 68, 035103(R) (2003)]

\title{
Epidemic Incidence in Correlated Complex Networks
}

\author{
Yamir Moreno, ${ }^{1,2}$ Javier B. Gómez, ${ }^{3}$ and Amalio F. Pacheco ${ }^{1,2}$ \\ ${ }^{1}$ Departamento de Física Teórica, Universidad de Zaragoza, Zaragoza 50009, Spain \\ ${ }^{2}$ Instituto de Biocomputación y Física de Sistemas Complejos, \\ Universidad de Zaragoza, Zaragoza 50009, Spain \\ ${ }^{3}$ Departamento de Ciencias de la Tierra, Universidad de Zaragoza, Zaragoza 50009, Spain
}

(Dated: October 10, 2018)

\begin{abstract}
We introduce a numerical method to solve epidemic models on the underlying topology of complex networks. The approach exploits the mean-field like rate equations describing the system and allows to work with very large system sizes, where Monte Carlo simulations are useless due to memory needs. We then study the SIR epidemiological model on assortative networks, providing numerical evidence of the absence of epidemic thresholds. Besides, the time profiles of the populations are analyzed. Finally, we stress that the present method would allow to solve arbitrary epidemic-like models provided that they can be described by mean-field rate equations.
\end{abstract}

PACS numbers: 89.75.-k, 89.75.Fb, 05.70.Jk, 05.40.a

A few years ago, Watts and Strogatz [1] introduced a model able to produce networks with properties of both regular lattices and random graphs with small diameter. Their model soon led to a burst of activity in the field 2, 3], further spurred by Barabasi and collaborators who found that many seemingly diverse systems share several topological properties such as a power law behavior in their connectivity distributions when represented as networks [4]. These complex networks are formed by a set of many elements (or nodes) that are linked together through edges (or links) if they interact directly. Empirical evidence supports that in notable networks, such as metabolic or communication webs, the probability $P(k)$ that any node has $k$ links to other nodes is distributed accordingly to a power law $P(k) \sim k^{-\gamma}$ [5, 6, 7], with $\gamma \leq 3$ in most cases.

Networks of this type, called scale-free (SF) networks, show a noticeable property: the heterogeneity of the connectivity distribution can not be neglected. One of the fundamental results derived from this property is that the threshold characterizing the percolation transition or an epidemic outbreak is vanishing in the thermodynamic limit 8, 9, 10, 11]. On the other hand, many real-world networks are also characterized by degree correlations 12, 13, which make it necessary to reconsider the same problems but taking into account the conditional probability $P\left(k^{\prime} \mid k\right)$ that a link emanating from a node of connectivity (or degree) $k$ leads to a node of connectivity $k^{\prime}$. Very recently, it has been shown analytically that the presence of nontrivial correlations does not change the main conclusions drawn for uncorrelated graphs, namely, the absence of percolation and epidemic thresholds 14, 15] under very general conditions. However, neither complete exact solutions nor nu- merical studies of the system's dynamics have been reported. From a theoretical side, it seems an unsurmountable task to work out such study. In contrast, although feasible in principle, numerical simulations using Monte Carlo techniques would be quite arduous since one should first generate a network with the proper correlations and then perform expensive Monte Carlo simulations for large system sizes, where memory requirements could seriously limit the size of the networks under study.

In this paper, we present an efficient numerical method that allows the study of epidemic-like models on the underlying topology of complex networks with arbitrary connectivity distributions and degree-degree correlations. The method solves the mean-field rate equations describing the dynamics of the system, where the topological properties are accounted for. Specifically, we fully analyze the Susceptible-Infected-Removed (SIR) model in networks with assortative correlations, where nodes tend to be linked with their connectivity peers [16] as for the case of social networks. Our results are compared with those obtained for uncorrelated networks and confirm the previous findings about the absence of any epidemic threshold. Besides, we report on the time evolution of the individual populations which allow us to draw interesting conclusions when confronted to uncorrelated networks.

The SIR model 17, 18] considers that individuals are classified in three classes according to their state: susceptible, infected and removed. The epidemic is propagated by contacts between infected and susceptible individuals at a rate $\lambda$. Thus, once an individual gets infected and recovers he can not catch the epidemic again. Moreover, in networks with power-law distributed connectivities one also has to consider the presence of nodes with different connectivity $k$ within each category. We consider 
the time evolution of the magnitudes $\rho_{k}(t), s_{k}(t)$, and $r_{k}(t)$, which are the density of infected, susceptible, and removed nodes of connectivity $k$ at time $t$, respectively, with the normalization condition $\rho_{k}(t)+s_{k}(t)+r_{k}(t)=1$. Global quantities can be obtained by averaging over the connectivity classes. In this way, the fraction of infected individuals (or the epidemic incidence) is given by $r(t)=\sum_{k} P(k) r_{k}(t)$.

The mean-field rate equations for the evolution of these densities satisfy the following set of coupled differential equations [17]:

$$
\begin{aligned}
& \frac{d s_{k}(t)}{d t}=-\lambda k s_{k}(t) \sum_{k^{\prime}} P\left(k^{\prime} \mid k\right) \rho_{k^{\prime}}, \\
& \frac{d \rho_{k}(t)}{d t}=-\rho_{k}(t)+\lambda k s_{k}(t) \sum_{k^{\prime}} P\left(k^{\prime} \mid k\right) \rho_{k^{\prime}}, \\
& \frac{d r_{k}(t)}{d t}=\rho_{k}(t) .
\end{aligned}
$$

with the initial conditions $s(0)=(N-1) / N, \rho(0)=1 / N$ and $r(0)=0$. In the above equations, it is considered that infected individuals recover with unitary rate. Moreover, the creation term in Eq. (2) is proportional to the density of susceptible individuals, $s_{k}(t)$, times the spreading rate $\lambda$, the number of emanating links $k$ and the probability that any neighboring node is infected. This probability is given by the average over all degrees of the probability $P\left(k^{\prime} \mid k\right) \rho_{k^{\prime}}$ that a link emanated from a node with connectivity $k$ points to an infected node with degree $k^{\prime}$ [15, 19].

The numerical approach introduced here is based on a different interpretation of the mean-field rate equations (13) and is implemented as follows. Indeed, these equations describe a process in which individuals are decaying from one state to another. Hence, one can speak in terms of transition probabilities from the class of susceptible onto infected and finally onto removed individuals, i.e., $s_{k} \stackrel{W_{s \rho}^{k}}{\longrightarrow} \rho_{k} \stackrel{W_{\rho r}^{k}}{\longrightarrow} r_{k}$. From Eqs. (13) we get the transition probabilities at each time step $t$ :

$$
\begin{aligned}
& W_{s \rho}^{k}(t)=\lambda k N P(k) s_{k}(t) \sum_{k^{\prime}} P\left(k^{\prime} \mid k\right) \rho_{k^{\prime}}(t), \\
& W_{\rho r}^{k}(t)=N P(k) \rho_{k}(t),
\end{aligned}
$$

where all the topological information is contained. The mean time interval, $\tau$, for one transition to occur after $i-1$ decays is,

$$
\tau=\frac{1}{W_{s \rho}(t)+W_{\rho r}(t)},
$$

with $W_{s \rho}(t)=\sum_{k} W_{s \rho}^{k}(t), W_{\rho r}(t)=\sum_{k} W_{\rho r}^{k}(t)$ and $t=\sum_{j}^{i-1} \tau_{j}$. That is, at any instant $t$ of the decaying process, $\tau$ represents the mean time for the next individual decay. Once the transition probabilities (4.5)

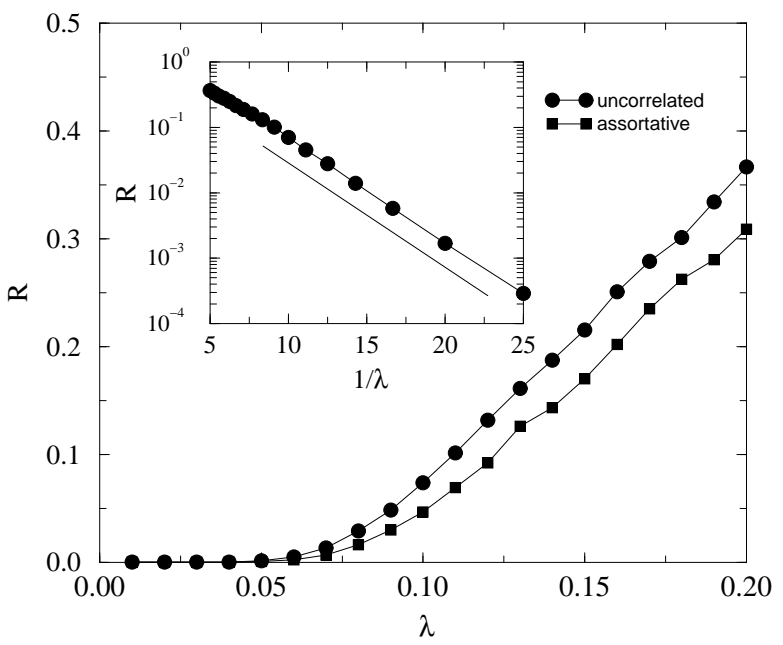

FIG. 1: Epidemic incidence in scale-free networks with $\gamma=3$ and $N=10^{5}$ with (full squares) and without correlations (full circles) as a function of the spreading rate $\lambda$. The assortative correlations are given by Eq. (9) with $\alpha=0.1$. The inset is a fit to the analytic relation $R \sim e^{-2 /\langle k\rangle \lambda}$. See the text for details.

are calculated, we stochastically decide what transition takes place. Nodes are divided in three classes according to their state and, within each of these classes, they are also characterized by their connectivity $k$. Hence, the identification of both what transition occurs and which class $k$ is affected after one $\tau$ is done by deciding that the probabilities that precisely a node with connectivity $k$ changes its state at time $t$ are given by

$$
\Pi_{s \rho}^{k}(t)=W_{s \rho}^{k}(t) \tau, \quad \Pi_{\rho r}^{k}(t)=W_{\rho r}^{k}(t) \tau,
$$

materializing the choice by generating a random number between 0 and 1 . In other words, the individual decays within each connectivity class $k$ proceeds by chance with the probabilities dictated by Eq. (7) until the end of the spreading process, $i . e$., when the condition $\rho_{k}=0$ is verified $\forall k$. It is worth mentioning that the present stochastic approach has been successfully applied to the study of time-dependent models of fracture [20, 21]. Besides, we remark that the main advantage of the method lies in the fact that we don't have to know explicitly the connectivity matrix, but only a functional form for $P\left(k^{\prime} \mid k\right)$. Therefore, in contrast to Monte Carlo simulations, we do not generate any network. Instead, we generate a sequence of integers distributed according to a power law $P(k) \sim k^{-\gamma}$ for the node's connectivities.

In order to compare the SIR dynamics in uncorrelated and correlated networks, we set henceforth $\gamma=3$. For uncorrelated networks, the two-point degree correlation function $P\left(k^{\prime} \mid k\right)$ is of the form

$$
P\left(k^{\prime} \mid k\right)=q_{k^{\prime}}=\frac{k^{\prime} P\left(k^{\prime}\right)}{\langle k\rangle} .
$$




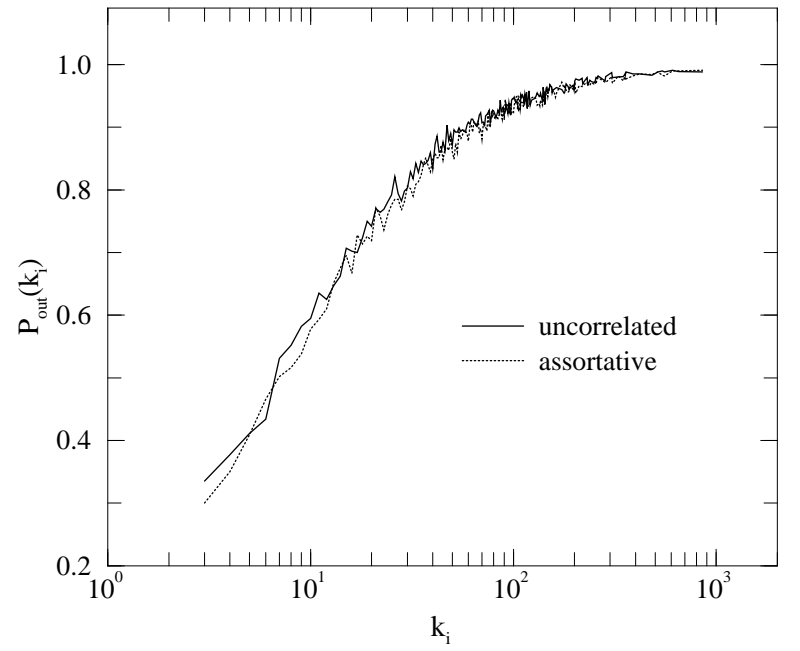

FIG. 2: Likelihood of an epidemic outbreak as a function of the connectivity of the initial seed. The value of $\lambda$ has been set to 0.15 . Note that the introduction of correlations does not change the probability of having an outbreak. The figure is in log-linear scales for clarity. The network's parameters are as of Fig. 1

Furthermore, consider the case in which the degree correlations can be decomposed into two components

$$
P\left(k^{\prime} \mid k\right)=(1-\alpha) q_{k^{\prime}}+\alpha \delta_{k k^{\prime}}
$$

with $0 \leq \alpha<1$. Varying the parameter $\alpha$ one interpolates between the uncorrelated graphs $(\alpha=0)$ and a graph with positive degree correlations 14, 22.

We have studied the effects introduced by assortative correlations given by Eq. (9) in the spreading of a disease. Social networks are the capital example of assortative networks and, although positively correlated, do not have high correlation coefficients [16], hence, we have used small values of $\alpha$ in our simulations. Figure 1 shows the epidemic incidence, $R=r\left(t_{\infty}\right)=\sum_{k} P(k) r_{k}\left(t_{\infty}\right)$, averaged over many realizations, where $t_{\infty}$ is the lifetime of an epidemic outbreak. The results indicate that the epidemic incidence in networks with assortative correlations is smaller that for uncorrelated ones with the same degree distribution. However, the epidemic threshold in the thermodynamic limit is not modified by the presence of positive correlations and is vanishing when $N \rightarrow \infty$. The inset in Fig. 1 is a plot of $R$ as a function of $1 / \lambda$ for an uncorrelated network with $\langle k\rangle=6$. The behavior nicely fits the theoretical prediction $R \sim e^{-2 /\langle k\rangle \lambda}$ [17], being the slope of the straight line equal to 0.36(1). This constitutes a further test of the validity of the approach presented here. As to the correlated networks, they satisfy the same functional form $R \sim e^{-C / \lambda}$ but approaches zero more slowly. Thus, the numerical findings confirm some theoretical arguments recently pointed out about the absence of any epidemic threshold in SF correlated

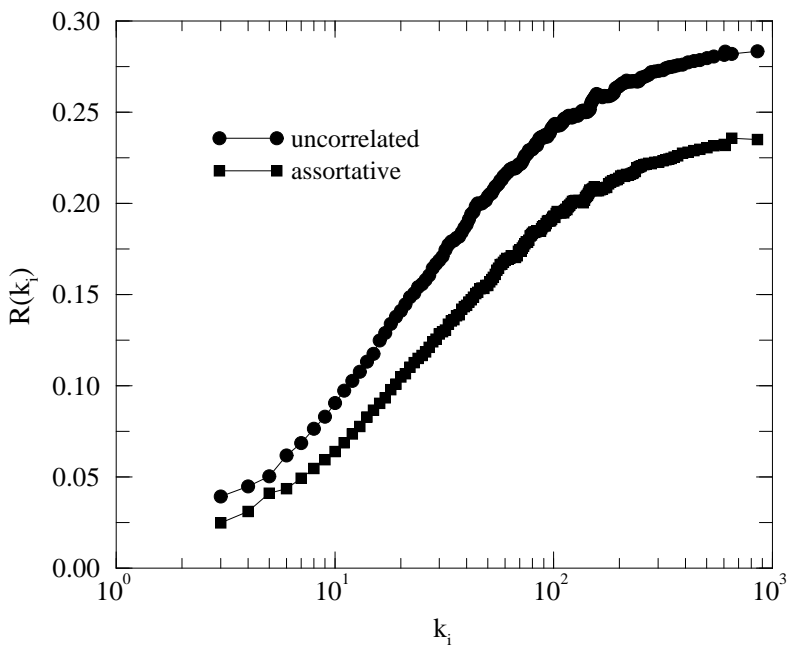

FIG. 3: Epidemic incidence as a function of the connectivity of the initial seed for $\lambda=0.15$. The dependency on the initial seed is stronger for uncorrelated networks. The network's parameters are as of Fig. 1 See the text for further details.

networks with $\gamma \leq 3[14,15]$. Moreover, we note that in finite size networks with assortative correlations the effective threshold is larger, suggesting that these networks are more robust than uncorrelated ones.

The high heterogeneity of SF networks also causes that the relative incidence of an outbreak strongly depends on the connectivity of the first infected nodes [17]. First, we explored the likelihood of an epidemic outbreak, $P_{\text {out }}$ as a function of the connectivity, $k_{i}$, of the initial infected node. This probability is obtained by dividing the number of times an epidemic developed by the total attempts made. The results are summarized in Fig. 2 As can be observed, no matter whether or not correlations are present, the probability of having an epidemic outbreak is the same for both kind of networks. This behavior changes when coarse-graining the results of Fig. 1 The results shown in Fig. [3 have been obtained by simulating the SIR dynamics when the initial infected node has a connectivity $k_{i}$ and recording the epidemic incidence in each case. Now, the number of individuals within the removed class at the end of the spreading process significantly depends on the correlations. Note that this is not a consequence of the smaller value of the epidemic incidence for assortative correlations. In fact, the first part of the curves, up to intermediate values of $k_{i}$, follows a logarithmic dependency on the connectivity, but the number of removed individuals for uncorrelated networks grows more faster than for correlated ones. This behavior can be intuitively understood since in assortative networks, nodes are mainly connected with their connectivity peers (even for small values of $\alpha$ ), whereas in uncorrelated networks it is always possible that a poorly connected node transmits the disease to a hub. In other 


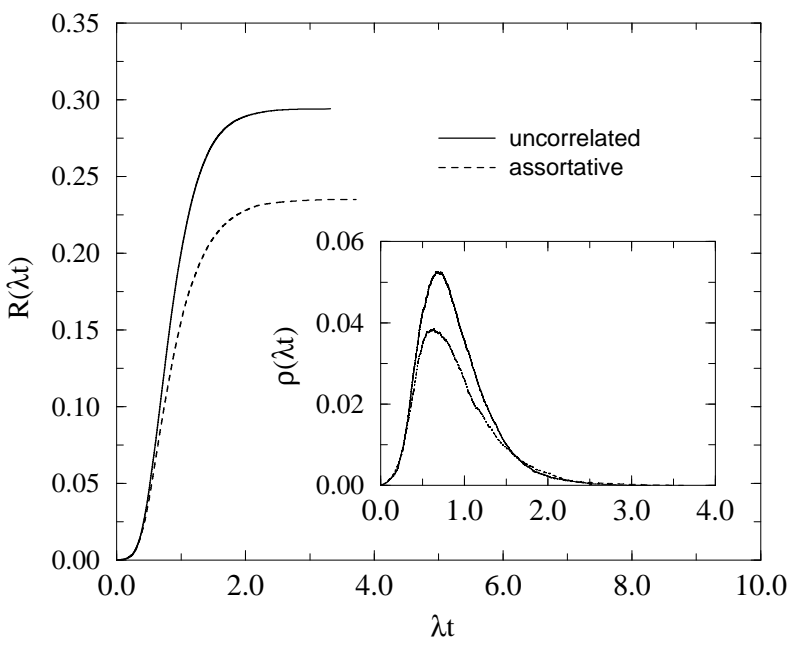

FIG. 4: Time evolution of the epidemic incidence (main figure) and of the density of infected individuals (inset) when the initial infected individual has the largest connectivity $k_{\max }$ for both uncorrelated and correlated networks. $\lambda$ is equal to 0.15 . The network's parameters are as of Fig [

words, the random mixing leads to a higher $R$ and favors the propagation of the outbreak.

The method introduced here also allows the exploration of the time dependency of the quantities characterizing the epidemic spreading. Note that the time profiles, in contrast to MC simulations, are in units of $\lambda^{-1}$ and thus they can be used as a real quantitative measure. Figure 4 plots the time evolution of the fraction of removed individuals and that of the infected nodes for the two networks under study when the initial infected individual has the largest connectivity $k_{\max }$. Here, as before, by simple inspection one would not be able to distinguish the behavior of these magnitudes in both networks as they show the same functional dependency on time. However, a more careful look at the plateau of $R(\lambda t)$ reveals that the epidemic lifetime is longer for assortative correlations than for uncorrelated networks. This is a direct consequence of the correlations. Starting from the very hub of the network, as time goes on, the epidemic is propagated, on average, from highly connected nodes down to less connected individuals such that when the end of the process is approaching, the mean time for individual decays is longer and longer leading to an effective deceleration of the epidemic spreading process. This is not anymore the case when random mixing rules out any correlated spreading. A further evidence of this mechanism is provided in the inset where it can be clearly noted that the two density profiles crosses well before the final death of the disease.

In summary, we have introduced an efficient numerical method that allows to explore the spreading of epidemic diseases in complex correlated networks without gener- ating explicitly the network with the proper correlations. We have studied the SIR epidemiological model in assortative networks and found that its qualitative behavior is the same as for uncorrelated networks. However, from a more practical perspective, there are some important quantitative differences that deserve to be considered more carefully and makes the inclusion of correlations in nowadays studies meaningful. In particular, while the likelihood of an epidemic outbreak is not modified when taking into account positive correlations, the epidemic incidence is smaller than in networks with no correlations. In large social networks this may lead to a difference of $15 \%$ to $20 \%$ of infected people for moderate values of $\lambda$. On the other hand, we have found that the diseases are longest-lived in assortative networks. Additionally, we stress that the method employed here can be used to solve other epidemic-like models in networks with any correlations such as the SIS 11] and rumor spreading 23. models, provided that they can be described through mean-field rate equations. Finally, we point out that results for disassortative networks will appear elsewhere.

Y. M. thanks A. Vázquez for useful discussions. Y. M. acknowledges financial support from the Secretaría de Estado de Educación y Universidades (Spain, SB20000357). This work has been partially supported by the Spanish DGICYT project BFM2002-01798.

[1] D. J. Watts and H. S. Strogatz, Nature 393, 440 (1998).

[2] S. H. Strogatz, Nature (London) 410, 268 (2001).

[3] S. N. Dorogovtsev and J. F. F. Mendes, Adv. Phys. 51, 1079 (2002).

[4] A.-L. Barabási, and R. Albert, Science 286, 509 (1999).

[5] R. Albert and A.-L. Barabási, Rev. Mod. Phys. 74, 47 (2002).

[6] M. Faloutsos, P. Faloutsos, and C. Faloutsos, Comp. Com. Rev. 29, 251 (2000).

[7] R. Pastor-Satorras, A. Vázquez, and A. Vespignani, Phys. Rev. Lett. 87, 258701 (2001).

[8] R. Cohen, K. Erez, D. ben-Avraham, and S. Havlin, Phys. Rev. Lett. 85, 4626 (2000);

[9] D. S. Callaway, M. E. J. Newman, S. H. Strogatz, and D. J. Watts, Phys. Rev. Lett. 85, 5468 (2000).

[10] R. Albert, H. Jeong, and A.-L. Barabási, Nature 406, 378 (2000).

[11] R. Pastor-Satorras, and A. Vespignani, Phys. Rev. Lett. 86, 3200 (2001).

[12] M. E. J. Newman, Phys. Rev. Lett. 89, 208701 (2002).

[13] P. L. Krapivsky and S. Redner, Phys. Rev. E 63, 066123 (2001).

[14] A. Vázquez, and Y. Moreno, Phys. Rev. E 67, 015101(R) (2003).

[15] M. Boguñá, R. Pastor-Satorras and A. Vespignani, Phys. Rev. Lett. 90, 028701 (2003).

[16] M. E. J. Newman, Phys. Rev. E 67, 026126 (2003).

[17] Y. Moreno, R. Pastor-Satorras, and A. Vespignani, Eur. Phys. J. B 26, 521 (2002).

[18] M. E. J. Newman, Phys. Rev. E 66, 016128 (2002). 
[19] We assume that the conditions for the set of equations to be meaningful are satisfied [15].

[20] J. B. Gómez, Y. Moreno, and A. F. Pacheco, Phys. Rev. E 58, 1528 (1998).

[21] Y. Moreno, A. M. Correig, J. B. Gómez,and A. F.
Pacheco, J. Geophys. Res. B 106, 6609 (2001)

[22] A. Vázquez, and M. Weigt, Phys. Rev. E 67, 027101 (2003).

[23] Y. Moreno, M. Nekovee, and A. Vespignani, in preparation. 